\title{
NaF uptake in unstable plaque: what does fluoride uptake mean?
}

\author{
Takehiro Nakahara ${ }^{1} \cdot$ Jagat Narula ${ }^{2} \cdot$ H. William Strauss ${ }^{3}$ \\ Published online: 2 October 2018 \\ (C) Springer-Verlag GmbH Germany, part of Springer Nature 2018
}

Coronary artery calcification has been used to predict the risk of acute coronary events. Coronary calcification is usually quantified using EKG gated computed tomography (CT) [1, $2]$. Recent studies suggest that the predictive value of coronary calcification is not linearly related to risk. For example, a 31\% higher progression of coronary artery calcium was demonstrated in patients taking statin medication, despite a lower cardiac event rate [3]. Intravascular ultrasound (IVUS) and $\mathrm{CT}$ angiographic studies have demonstrated that although patients with acute coronary syndromes (ACS) had more noncalcified plaques on CT than stable angina patients [4], spotty calcification was frequently observed in the culprit plaques associated with acute events; larger calcific masses did not identify unstable lesions $[5,6]$. This observation may be due to the fact that the largest surface area with exchangeable calcium occurs in regions of acute necrosis, where the sizes of lesions are measured in microns. The spatial resolution of clinical CT is $\sim 1-2 \mathrm{~mm}$, to limit patient radiation burden. Exvivo studies, on the other hand, where spatial resolution is $\sim 1 \mu \mathrm{m}$, demonstrated the presence of microcalcification in lesions that are not seen on clinical CT, but are associated with eventful plaques [7].

${ }^{18} \mathrm{~F}$-sodium fluoride positron emission tomography $(\mathrm{NaF})$ imaging, originally proposed as an agent to identify bone metastasis over 50 years ago [8], has been suggested as an agent to detect microcalcification in atherosclerotic plaques [9-11]. Although spatial resolution of molecular imaging is low, the

This Editorial Commentary refers to the article https://doi.org/10.1007/ s00259-018-4122-0.

\section{H. William Strauss}

harry.strauss@gmail.com

1 Department of Diagnostic Radiology, Keio University School of Medicine, 35 Shinanomachi, Shinjuku-ku, Tokyo, Japan

2 Division of Cardiology, Icahn School of Medicine at Mount Sinai, New York, NY, USA

3 Molecular Imaging and Therapy Service, Memorial Sloan Kettering Cancer Center, New York, NY, USA high affinity of $\mathrm{NaF}$ for microcalcification allows visualization of very small lesions.

In this issue of the EJNMMI, Li et al. [12] describe the relationship between ${ }^{18} \mathrm{~F}-\mathrm{NaF}$ coronary uptake on PET/ $\mathrm{CT}$, to coronary IVUS-verified high-risk plaque characteristics and coronary artery calcium distribution based on Electron Beam CT calculation of Agatston score, calcium mass and calcium volume. Li et al. studied 32 symptomatic patients, including 30 with unstable angina and two with stable angina. The investigators used high-frequency IVUS (40 MHz, pullback: $0.5 \mathrm{~mm} / \mathrm{s}$ ) with radiofrequency analysis (Virtual Histology, VH) for target lesions as well as proximal and distal reference segments to characterize coronary plaques. Electrocardiogram-gated ${ }^{18}$ F-PET-CT was performed within 2 days before IVUS. Sixty minutes after IV administration of ${ }^{18} \mathrm{~F}-\mathrm{NaF}$, a 10 min gated, static, PET CT was recorded in one bed position, covering the heart and the aortic arch. Two-dimensional regions of interest (ROI) (diameter: 1-2 mm) were manually set on the target lesion to measure maximum standardized uptake value (SUV max). As a background, the average SUVmax values of three ROIs in the superior vena cava were measured. The lesion SUVmax was divided by the vena cava (SUVblood) to calculate the maximum tissueto-blood (TBRmax) ratio. The authors set TBRmax of 1.25 as a threshold cut-off value. A total of 69 coronary atherosclerotic lesions were evaluated. NaF uptake ratio was increased in thin-cap atheroma with spotty calcification, thick-cap atheroma and fibrocalcific lesions, but not increased in fibrous plaque. $\mathrm{NaF}$ uptake correlated with the severity of plaque burden, positive lesion remodeling, and the extent of necrotic core, but was negatively correlated with fibrotic component of the plaque. In a subgroup analysis of 128 arteries (LMCA, LAD, LCx, and RCA) in the 32 patients grouped by Agatston score, there was no correlation of NaF uptake with calcium burden on EBCT.

High-risk plaques are characterized by: large plaque burden with necrotic core, significant infiltration with cells of monocyte-macrophage lineage, increased density of vasa vasorum, and microcalcification [7]. Atheroma are initiated 
by sub-intimal insudation of LDL cholesterol, followed by infiltration of inflammatory cells to phagocytize the LDL cholesterol, leading to the formation of cholesterol-laden foam cells. The inflamed atheroma is hypoxic, resulting in local release of factors to promote the development of additional vasa vasorum. Early in the life history of an atheroma, the foam cells die by apoptosis and the remnants of the apoptotic cells are phagocytized by surrounding macrophages. In more advanced lesions, inefficient efferocytosis causes apoptotic debris to accumulate, resulting in expansion of the necrotic core and further inflammation. The inflammatory milieu results in collagenolysis and renders the plaque unstable. Microcalcification is initiated in regions of inflammation where cell death is abundant and provides a nidus for calcium seeding [13]. The microcalcific deposits evolve to spotty calcification (SC) easily recognized on CT. If the inflammation is reduced (usually by medication and diet), the lesions stabilize with elimination of foam cells and massive cell death leads to macrocalcification or plate-like calcification (PC) [7, 14-16]. Therefore, micro or spotty calcification or SC may identify atrisk lesions but when extensive (PC), may represent plaque stability. The statin-related calcific progression represents the latter phenomenon. It is therefore important to identify the calcification process rather than calcific fossils that may support the importance of $\mathrm{NaF}$ imaging.

The authors have described four types of $\mathrm{NaF}$ uptake in plaques and we should try to reconcile the uptake based on the foregoing molecular mechanisms. High $\mathrm{NaF}$ uptake of type 1 (thick-cap fibroatheroma with dense necrotic tissue) and type 2 (thin-cap fibroatheroma with dense necrotic tissue) plaques and low uptake of type 3 (fibrotic plaque poor necrotic tissue) seem reasonable. It is also understandable that $\mathrm{NaF}$ uptake did not correlate with calcium score but correlated with circulating levels of high sensitivity CRP.

The patients with type 4 fibrocalcific plaques with intensive calcification also showed high $\mathrm{NaF}$ uptake, which is more challenging to explain. It might alert us that a simple CTAbased notion of dangerous SC but a guarantee of plaque stability from PC may not be entirely justified. It is hypothetically reasonable to propose that $\mathrm{NaF}$ imaging might have the ability to discriminate macrocalcification with or without the inflammatory milieu and hence evil and not-so-evil plaques. On the other hand, it is also possible that $\mathrm{NaF}$ uptake seen with extensive CT-verified calcification may mean progression of calcification rather than inflammatory calcification and that $\mathrm{NaF}$ uptake in the presence of PC may represent a benign phenomenon. It is also possible that this unexpected finding may be due to the patient population and/or the limited number of patients investigated. This important question could be answered by a multi-center prospective case study to understand the clinical significance of scans that are: $\mathrm{PC}+\mathrm{NaF}+$, $\mathrm{PC}+\mathrm{NaF}-, \mathrm{PC}-\mathrm{NaF}+, \mathrm{PC}-\mathrm{NaF}-\mathrm{SC}+\mathrm{NaF}+, \mathrm{SC}-\mathrm{NaF}+, \mathrm{SC}+$ $\mathrm{NaF}-$, and $\mathrm{SC}-\mathrm{NaF}-$. It is also necessary that we reassess the possibility of NaF uptake by other cells [17] that might substantially alter our understanding of clinical implications of predictive, preventive or punitive roles of calcific deposits in the plaques [18]. Calcification is ubiquitous in atherosclerosis and hence its contribution to the plaque behavior and prognostic outcomes needs careful characterization.

\section{References}

1. Agatston AS, Janowitz WR, Hildner FJ, Zusmer NR, Viamonte M Jr, Detrano R. Quantification of coronary artery calcium using ultrafast computed tomography. J Am Coll Cardiol. 1990;15:827-32.

2. Mark DB, Berman DS, Budoff MJ, et al. ACCF/ACR/AHA/ NASCI/SAIP/SCAI/SCCT 2010 expert consensus document on coronary computed tomographic angiography: a report of the American College of Cardiology Foundation task force on expert consensus documents. J Am Coll Cardiol. 2010;55:2663-99.

3. Dykun I, Lehmann N, Kalsch H, et al. Statin medication enhances progression of coronary artery calcification: the Heinz Nixdorf recall study. J Am Coll Cardiol. 2016;68:2123-5.

4. van Velzen JE, de Graaf FR, Jukema JW, et al. Comparison of the relation between the calcium score and plaque characteristics in patients with acute coronary syndrome versus patients with stable coronary artery disease, assessed by computed tomography angiography and virtual histology intravascular ultrasound. Am J Cardiol. 2011;108:658-64.

5. Mauriello A, Servadei F, Zoccai GB, et al. Coronary calcification identifies the vulnerable patient rather than the vulnerable plaque. Atherosclerosis. 2013;229:124-9.

6. Otsuka F, Sakakura K, Yahagi K, Joner M, Virmani R. Has our understanding of calcification in human coronary atherosclerosis progressed? Arterioscler Thromb Vasc Biol. 2014;34:724-36.

7. Nakahara T, Dweck MR, Narula N, Pisapia D, Narula J, Strauss HW. Coronary artery calcification: from mechanism to molecular imaging. J Am Coll Cardiol Img. 2017;10:582-93.

8. Blau M, Nagler W, Bender MA. Fluorine-18: a new isotope for bone scanning. J Nucl Med. 1962;3:332-4.

9. Derlin T, Richter U, Bannas P, et al. Feasibility of $18 \mathrm{~F}$-sodium fluoride PET/CT for imaging of atherosclerotic plaque. J Nucl Med. 2010;51:862-5.

10. Dweck MR, Chow MW, Joshi NV, et al. Coronary arterial $18 \mathrm{~F}$ sodium fluoride uptake: a novel marker of plaque biology. J Am Coll Cardiol. 2012;59:1539-48.

11. Irkle A, Vesey AT, Lewis DY, et al. Identifying active vascular microcalcification by (18)F-sodium fluoride positron emission tomography. Nat Commun. 2015;6:7495.

12. Li L, Li X, Jia Y, Fan J, Wang H, Fan C, et al. Sodium-fluoride PET$\mathrm{CT}$ for the non-invasive evaluation of coronary plaques in symptomatic patients with coronary artery disease: a cross-correlation study with intravascular ultrasound. Eur J Nucl Med Mol Imaging. 2018. https://doi.org/10.1007/s00259-018-4122-0.

13. Hutcheson JD, Goettsch C, Bertazzo S, et al. Genesis and growth of extracellular-vesicle-derived microcalcification in atherosclerotic plaques. Nat Mater. 2016;15:335-43.

14. Nakahara T, Narula J, Strauss HW. Molecular imaging of vulnerable plaque. Semin Nucl Med. 2018;48:291-8.

15. Nakahara T, Strauss HW. From inflammation to calcification in atherosclerosis. Eur J Nucl Med Mol Imaging. 2017;44:858-60.

16. Nakahara T, Narula J, Strauss HW. Calcification and inflammation in atherosclerosis: which is the chicken, and which is the egg? J Am Coll Cardiol. 2016;67:79-80. 
17. Gutowska I, Baranowska-Bosiacka I, Siennicka A, Rybick M, Baśkiewicz M, Machaliński B, et al. Fluoride effects on cholesterol influx and expression of the scavenger receptor CD36 in THP1 differentiated monocyte/macrophage cells. Research report. Fluoride. 2011;44:135-42.
18. Shaw LJ, Narula J, Chandrashekhar Y. The never-ending story on coronary calcium: is it predictive, punitive, or protective? J Am Coll Cardiol. 2015;65:1283-5. 